\title{
MicroRNA-200c regulates cisplatin resistance by targeting ZEB2 in human gastric cancer cells
}

\author{
TAO JIANG ${ }^{1,2}$, PENGFEI DONG ${ }^{2},{\text { LONG } \mathrm{LI}^{2}, \text { XIAO MA }}^{2}$, PEI XU ${ }^{2}, \mathrm{HE} \mathrm{ZHU}^{2}$, YANQIU WANG ${ }^{2}$, \\ BAOTONG YANG ${ }^{2}$, KUANGGE LIU $^{2}$, JINWEI LIU ${ }^{2}$, JUAN XUE ${ }^{2}$, RUNZHE LV $^{2}$, PANKE SU ${ }^{2}$, \\ GUOQIANG KONG ${ }^{2}$, YONGCHAO CHANG ${ }^{2}$, CHONGGAO ZHAO ${ }^{2}$ and LIDONG WANG ${ }^{1}$ \\ ${ }^{1}$ Henan Key Laboratory - Esophageal Cancer Laboratory for Cancer Research, Basic Medical College, \\ Zhengzhou University, Zhengzhou, Henan 450052; ${ }^{2}$ Department of Clinical Laboratory, The First Affiliated Hospital \\ of Henan University of Science and Technology, Luoyang, Henan 471013, P.R. China
}

Received July 27, 2016; Accepted September 9, 2016

DOI: $10.3892 /$ or.2017.5659

\begin{abstract}
This study was specifically designed to confirm the hypothesis that microRNA-200c (miR-200c) affects the development of cisplatin (DDP) resistance in human gastric cancer cells by targeting zinc finger E-box binding homeobox 2 (ZEB2). A total of 50 gastric cancer tissues and their corresponding normal adjacent tissue samples were collected. Then, the expression levels of miR-200c and ZEB2 in both gastric cancer specimens and cells were detected using the quantitative real-time reverse transcription-polymerase chain reaction (qRT-PCR) and immunohistochemical methods. A dual-luciferase reporter gene assay was conducted to evaluate the effect of miR-200c on the 3'-untranslated region (3'UTR) luciferase activity of ZEB2. SGC7901/DDP cells were transfected with miR-200c mimics and ZEB2 siRNA, respectively. Subsequently, changes in cellular proliferation and apoptosis were detected through the methyl thiazolyl tetrazolium assay and flow cytometric analysis, respectively. We also carried out a western blot analysis assay in order to detect the expression of apoptosis-related genes and ZEB2. miR-200c was significantly downregulated and ZEB2 was significantly upregulated in both gastric cancer tissues and SGC7901/DDP cells when compared with those in normal tissues and SGC7901 cells $(\mathrm{P}<0.01)$. The dual luciferase reporter gene assay showed that miR-200c could specifically bind with the 3'UTR of ZEB2 and significantly suppress the luciferase activity by $42 \%(\mathrm{P}<0.01)$. Upregulation of $\mathrm{miR}-200 \mathrm{c}$ or downregulation of ZEB2 enhanced the sensitivity of SGC7901/DDP cells to DDP. miR-200c was significantly downregulated in both gastric cancer tissues and cells, while the expression of ZEB2
\end{abstract}

Correspondence to: Dr Lidong Wang, Henan Key Laboratory Esophageal Cancer Laboratory for Cancer Research, Basic Medical College, Zhengzhou University, 1 Jianshe East Road, Zhengzhou, Henan 450052, P.R. China

E-mail: anglilivv@yeah.net

Key words: gastric cancer, microRNA-200c, zinc finger E-box binding homeobox 2, cisplatin resistance, apoptosis exhibited the opposite trend. Our study further demonstrated that miR-200c could enhance the sensitivity of SGC7901/DDP cells to DDP through targeted regulation of ZEB2 expression in gastric cancer tissues.

\section{Introduction}

Gastric cancer is the most common gastrointestinal malignant tumor in the world and a large number of factors that have significant long-term impact have been linked with this disease (1). The morbidity and mortality for gastric cancer appears to be 14.1/100,000 and 10.3/100,000 cases, respectively (2). Numerous studies have shown that Helicobacter pylori infection is a major risk factor for gastric cancer since persistent Helicobacter pylori infections may consequently cause damage of the gastric mucosa and thereby trigger the proliferation of gastric epithelial cells. Moreover, Helicobacter pylori infection is also associated with the decrease in gastric acid secretion which may induce chronic gastritis, gastric precancerous lesions and gastric carcinogenesis (3). Other factors including family history, poor diet, smoking, drinking and mental stress are also involved in the development of gastric cancer $(1,4)$. Medications are still one of the most effective treatment options for patients with gastric cancer and they are usually recommended for patients in advanced stages (5). However, chemotherapeutic agents are not capable of effectively controlling the disease in a large proportion of patients as they present with resistance to these agents. One of the major limitations of chemotherapy may arise from the fact that some gastric cancer patients exhibit resistance to multiple medications and hence chemotherapy may not achieve its effectiveness (6). Therefore, a large amount of research has been conducted to overcome this issue.

MicroRNAs (miRNAs) are a conserved family of small non-coding RNA molecules that function as pivotal regulators of gene expression (7). They modulate gene expression by binding to the 3'-untranslated region (3'UTR) of their target mRNAs and thereby suppression of protein translation or mRNA decay can be triggered $(8,9)$. Furthermore, miRNAs not only participate in tumor proliferation, invasion and metastasis but also regulate a variety of biological 
processes (10-12). For instance, the expression of miR-200b/c in gastric cancer SGC7901/VCR cells was lower compared to that in SGC7901 cells whereas the expression of anti-apoptotic protein Bcl-2 was increased in SGC7901/VCR cells and there was a marked difference in its sensitivity to cisplatin (DDP) and vincristine (13). MicroRNA-200c (miR-200c) is part of the miR-200 family in which another four members are also included: miR-200a, miR-200b, miR-141 and miR-429 (14). In addition, the expression of miR-200c is significantly downregulated in tumor cells and tissues and it may play a role in tumor suppression $(15,16)$. For instance, Chen et al demonstrated that the expression of miR-200c was significantly lower in SGC7901/DDP cells whereas the sensitivity of SGC7901/DDP cells to four chemotherapy medications was significantly enhanced after the upregulation of miR-200c (17).

Zinc finger E-box binding homeobox 2 (ZEB2) is an important member of the Snail superfamily and it is commonly expressed in human and rat tissues. ZEB2 can bind the E-box sequence in the E-cadherin gene promoter region which inhibits E-cadherin, cytokeratin, mucin and macl protein transcription. More importantly, the downregulation of the aforementioned proteins plays an important role in epithelialto-mesenchymal transition (EMT) (18). Apart from that, ZEB2 is closely linked with bladder cancer resistance to epidermal growth factor inhibitor and ovarian cancer resistance to $\operatorname{DDP}(19,20)$.

No current evidence has been revealed with respect to the hypothesis that miR-200c regulates DDP resistance by targeting ZEB2 in human gastric cancer cells. As a result of this, we are motivated to systematically clarify the potential function of the miR-200c and ZEB2 signaling pathway in gastric cancer and DDP resistance may be predicted based on the aforementioned functions.

\section{Materials and methods}

Tissue samples. Fifty gastric cancer tissues and paired normal adjacent tissues were acquired from patients who underwent surgical treatment in our hospital from 2013 to 2015. There were 28 males and 22 females, with an average age of 55.6 years (ranging from 30 to 81 years). Clinical TNM stages were determined according to the newly revised standards of TNM staging for gastric cancer from the 2010 American Joint Committee on Cancer (AJCC) (21). No patients received radiotherapy, chemotherapy or hormone therapy before surgery. Tissue samples were immediately frozen in liquid nitrogen at the time of surgery, then stored at $-80^{\circ} \mathrm{C}$ until the extraction of RNA. Another group of tumor tissues was fixed with $10 \%$ formalin, embedded in paraffin and immunohistochemistry was performed. Informed consent for this study was collected from every patient, and this study was approved by the Research Ethics Committee of Henan Key Laboratory for Esophageal Cancer Laboratory for Cancer Research.

Cell culture. SGC7901, a human gastric cancer cell line, was obtained from the Cell Bank of the Chinese Academy of Sciences (Shanghai, China). The SGC7901/DDP cells with DDP resistance were purchased from KeyGen Biotech Co., Ltd. (Nanjing, China). Cells were maintained in RPMI-1640 medium containing $10 \%$ fetal bovine serum (FBS), streptomycin $(100 \mu \mathrm{g} / \mathrm{ml})$ and penicillin $(100 \mathrm{U} / \mathrm{ml})$ (all from Gibco, Grand Island, NY, USA) in a $37^{\circ} \mathrm{C}$ incubator with $5 \%$ $\mathrm{CO}_{2}$. In order to maintain the DDP resistant phenotype, DDP (at a final concentration of $1 \mu \mathrm{g} / \mathrm{ml}$ ) was added to the culture medium for the SGC7901/DDP cells. SGC7901/DDP cells were cultured for one week in medium without DDP before experimentation.

Cell transfection. We transfected miR-200c mimics and ZEB2 siRNA (purchased from GenePharma, Shanghai, China) into gastric cancer cells in order to assess the effect of miR-200c and ZEB2 on both cell apoptosis and chemosensitivity. The scramble group transfected with scramble miRNA mimics was synthesized by GenePharma and was used as the negative control. Cells were plated in 6-well plates at $1 \times 10^{5}$ cells in each well in an antibiotic-free RPMI-1640 medium with 10\% FBS. After 12 h, Opti-MEM medium (Gibco) without antibiotics and serum was used to replace the aforementioned medium. When cells grew to about $50 \%$ confluence, they were transfected with Lipofectamine 2000 (Invitrogen, Carlsbad, CA, USA) and cultured in a $37^{\circ} \mathrm{C}$ incubator with $5 \% \mathrm{CO}_{2}$. Cells continued to culture after the complete medium was replaced after 6-8 h. Cells were harvested after a 48-h transfection and used for western blot analysis and RT-PCR.

Quantitative real time reverse transcription-polymerase chain reaction $(q R T-P C R)$ analysis. Total RNA extraction from human gastric cancer tissues and cells was conducted using TRIzol reagent (Invitrogen) based on the manufacturer's instructions. Complementary DNA (cDNA) was acquired using the Omniscript reverse transcription kit (Qiagen, Hilden, Germany). After the reaction of reverse transcription, a real-time quantitative RT-PCR assay was conducted using an ABI7500 quantitative PCR instrument (Applied Biosystems) and the relative expression levels of miR-200c and ZEB2 mRNA were detected. The primers for miR-200c and ZEB2 (Invitrogen) are shown in Table I. The relative expression of miR-200c and ZEB2 mRNA was calculated using the $2^{-\Delta \Delta C t}$ method and normalized to the expression of U6 snRNA and glyceraldehyde-3-phosphate dehydrogenase (GAPDH). All the aforementioned assays were replicated three times.

Immunohistochemistry. ZEB2 protein expression in gastric cancer specimens was detected using the universal PV-9000 two-step immunohistochemical method. In brief, formalin-fixed and paraffin-embedded tissues from gastric cancer patients were cut into $4-\mu \mathrm{m}$ slices. Then, conventional dewaxing, graded ethanol dehydration, antigen retrieval and the addition of $3 \%$ hydrogen peroxide were performed on tissue slices in order to block endogenous peroxidase. Primary antibodies (rabbit anti-human ZEB2 polyclonal antibody; BIOSS, Beijing, China) were applied to tissue slices at $4^{\circ} \mathrm{C}$ overnight. Then, tissues were incubated for $20 \mathrm{~min}$ at room temperature after the addition of polymerase adjuvants. Secondary antibodies labeled with horseradish peroxidase (HRP; BIOSS) were also applied and tissues were incubated for another $30 \mathrm{~min}$ at room temperature. Staining was performed using diaminobenzidine (DAB) and slices were counterstained using hemalum. Phosphate-buffered solution (PBS) instead of a primary antibody was considered as the negative control 
Table I. Sequence of the primers used for quantitative RT-PCR.

Genes

Primer pair sequences

$\begin{array}{lll}\text { miR-200c } & \text { F: 5'-AGCGGTAATACTGCCGGGTA-3' } & \text { R: 5'-GTGCAGGGTCCGAGGT-3' } \\ \text { U6 } & \text { F: 5'-GGGTGCTCGCTTCGGCAGC-3' } & \text { R: 5'-CAGTGCAGGGTCCGAGGT-3' } \\ \text { ZEB2 } & \text { F: 5'-TGTCATTAGAAGAGGCGTAA-3' } & \text { R: 5'-GCAGAGCAGGTTAGAACT-3' } \\ \text { GAPDH } & \text { F: 5'-CATCAGCAATGCCTCCTGCAC-3' } & \text { R: 5'-TGAGTCCTTCCACGATACCAAAGTT-3' }\end{array}$

RT-PCR, reverse transcription-polymerase chain reaction; miR-200c, microRNA-200c; ZEB2, zinc finger E-box binding homeobox 2; GAPDH, glyceraldehyde-3-phosphate dehydrogenase; F, forward; R, reverse.

and a known positive antibody was set as the positive control. The immunohistochemical score of ZEB2 was calculated by multiplying the intensity of the staining ( 0 , colorless; 1, light yellow; 2, yellow; and 3, brown) and the positive cell percentage $(0, \leq 5 \% ; 1,6-25 \% ; 2,26-50 \% ; 3,51-75 \%$; and 4 , $>75 \%$ ). Cells were randomly selected from 5 high-power fields (x200) in each slice and 100 cells were counted in each field. As suggested by the final scores, the integral levels of ZEB2 were evaluated as: negative $(-), \leq 4$ points and positive $(+)$, $>4$ points. Two independent pathologists were responsible for analyzing these slices.

Dual-luciferase reporter assay. miRNA targeted genes were predicted using TargetScan (https://www.targetscan.org). Then, the wild-type and mutant-type of ZEB2 3'UTR luciferase reporter vectors were constructed. miR-200c mimics were co-transfected with constructed wild-type or mutant-type luciferase reporter vectors into SGC7901/DDP cells using Lipofectamine 2000 (Invitrogen). The pGL3-control vector (Promega, Madison, WI, USA) was transfected as a control. The dual-luciferase reporter assay system (Promega) was used to examine the luciferase activity after the cells had been transfected for $48 \mathrm{~h}$.

Cell viability assay. Cell viability was evaluated using the methyl thiazolyl tetrazolium (MTT) assay. Briefly, transfected cells were seeded in a 96-well plate at $5 \times 10^{3}$ cells in each well for $48 \mathrm{~h}$. After $12 \mathrm{~h}$, the cells were treated with various concentrations of DDP (Qilu Pharmaceutical Co., Ltd., Shandong, China) with final concentrations at $0.01,0.1,1$ and 10 times those of the human peak serum doses for DDP, as previously suggested (13). The peak plasma concentration of anticancer drugs is $2.0 \mu \mathrm{g} / \mathrm{ml}$ for DDP (22). Approximately $48 \mathrm{~h}$ after the addition of DDP, MTT (20 $\mu 1,5 \mathrm{mg} / \mathrm{ml}$; Sigma-Aldrich, St. Louis, MO, USA) was added into each well, and the culture was sustained for $4 \mathrm{~h}$ in a $37^{\circ} \mathrm{C}$ incubator with $5 \% \mathrm{CO}_{2}$. Subsequently, dimethyl sulphoxide (DMSO; $150 \mu \mathrm{l}$ ) was added into each well and the cells were shaken lightly for $10 \mathrm{~min}$ to dissolve the crystals. Samples were read on a microplate reader (SpectraMax Plus; Molecular Devices, Sunnyvale, CA, USA) at $490 \mathrm{~nm}$. The $50 \%$ inhibition concentration $\left(\mathrm{IC}_{50}\right)$ of DDP was estimated.

Apoptosis assay. Annexin V-fluorescein isothiocyanate (Annexin V-FITC) and propidium iodide (PI) apoptosis detection kit (Becton-Dickinson, Franklin Lakes, NJ, USA)

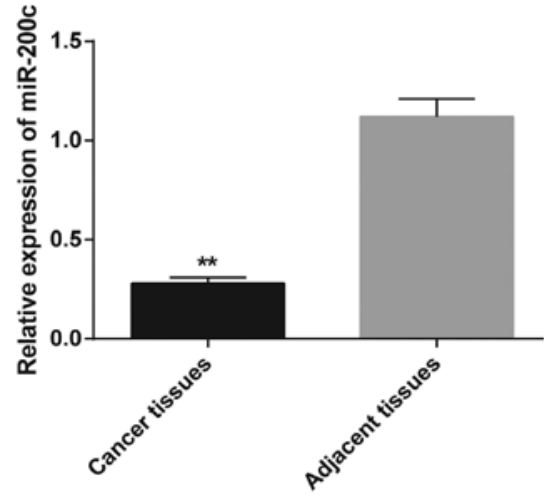

Figure 1. Relative expression of miR-200c in gastric cancer clinical specimens was detected by quantitative real-time RT-PCR. Data are expressed as the mean $\pm \mathrm{SD} .{ }^{* *} \mathrm{P}<0.01$, compared with the corresponding control group. miR-200c, microRNA-200c.

was used to evaluate the apoptosis of SGC7901/DDP cells. In brief, SGC7901/DDP cells were treated with DDP at a final concentration of $5 \mu \mathrm{g} / \mathrm{ml}$ after transfection for $24 \mathrm{~h}$. Cells following a 48-h treatment were washed two times using cold PBS. Then the cells were re-suspended in binding buffer and maintained at a concentration of $0.5-1 \times 10^{6} / \mathrm{ml}$. The suspension (100 $\mu \mathrm{l}$ ) was incubated with $5 \mu \mathrm{l}$ of Annexin V-FITC and PI for $15 \mathrm{~min}$ at room temperature in the dark. After the addition of $400 \mu \mathrm{l}$ binding buffer into each tube, the cells were assessed using flow cytometry (Beckman FC 500 MCL/MPL; Beckman Coulter, Brea, CA, USA).

Western blot analysis assay. After transfection was sustained for $48 \mathrm{~h}$, cells were collected and homogenized using RIPA buffer (Beyotime, China). Cellular proteins were extracted and the protein concentrations were assessed using a bicinchoninic acid (BCA) protein assay kit (Boster Biotechnology Co., Ltd., Wuhan, China). Equal amounts of proteins for each group were loaded and isolated with sodium dodecyl sulphatepolyacrylamide gel electrophoresis (SDS-PAGE), after which they were transferred onto polyvinylidene fluoride membranes and blocked with 5\% nonfat milk. Membranes were incubated with the ZEB2, Bcl-2, Bax, caspase-3 primary antibodies or the GAPDH antibody [Cell Signaling Technology (CST), Beverly, MA, USA] respectively, at $4^{\circ} \mathrm{C}$ overnight. Membranes were washed three times using Tris hydroxymethyl aminoethane (TBST; 10 min each) and then HRP-linked secondary antibodies were added followed by incubation at room temper- 
Table II. Expression of miR-200c in gastric cancer specimens and the correlation with the clinicopathological features.

\begin{tabular}{lccr}
\hline $\begin{array}{l}\text { Clinicopathological } \\
\text { factors }\end{array}$ & $\mathrm{N}$ & miR-200c & P-value \\
\hline $\begin{array}{l}\text { Age (years) } \\
\geq 55\end{array}$ & 26 & $0.29 \pm 0.03$ & 0.245 \\
$<55$ & 24 & $0.28 \pm 0.03$ & \\
Gender & & & \\
$\quad$ Male & 28 & $0.28 \pm 0.02$ & 0.254 \\
Female & 22 & $0.29 \pm 0.04$ & \\
Lymph node metastasis & & & \\
$\quad$ Yes & 34 & $0.27 \pm 0.02$ & $<\mathbf{0 . 0 0 1}$ \\
$\quad$ No & 16 & $0.31 \pm 0.02$ & \\
TNM stage & & & \\
I/II & 19 & $0.30 \pm 0.03$ & $<\mathbf{0 . 0 0 1}$ \\
III/IV & 31 & $0.27 \pm 0.02$ & \\
\hline
\end{tabular}

Bold indicates a significant difference. miR-200c, microRNA-200c.

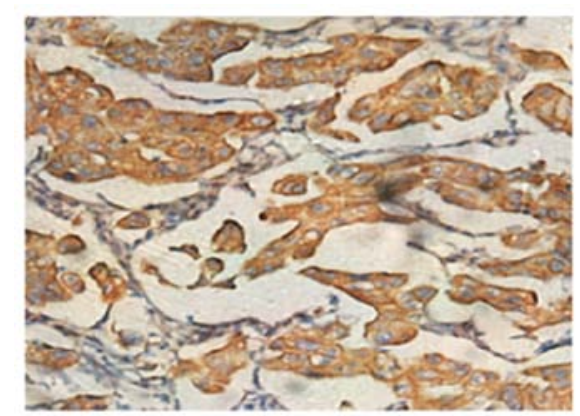

Adjacent normal tissues

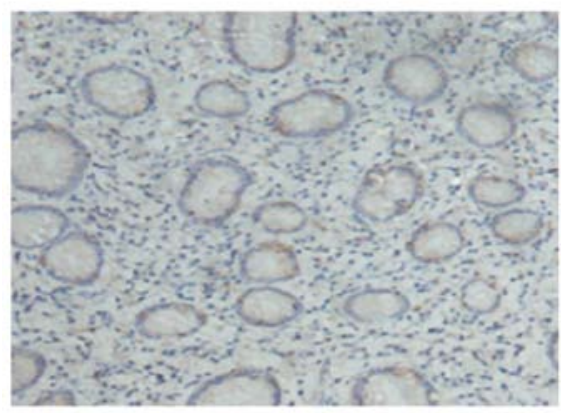

Gastric cancer tissues

Figure 2. ZEB2 expression in cancer tissues and adjacent tissues was detected by immunohistochemistry. The results were obtained from 3 independent experiments. Data are expressed as the mean $\pm \mathrm{SD}$. ${ }^{* *} \mathrm{P}<0.01$, compared with the corresponding control group. ZEB2, zinc finger E-box binding homeobox 2.

ature for $1 \mathrm{~h}$. Membranes were washed again with TBST three times (10 min each) and signal detection was performed using a Super ECL Plus Detection reagent (Applygen Technologies, Inc., Beijing, China).

Statistical analysis. Statistical analyses were implemented using the SPSS 19.0 software. Differences in continuous
Table III. Correlation between the expression of ZEB2 protein and the clinicopathological features of gastric cancer.

\begin{tabular}{lrrrr}
\hline & \multicolumn{4}{c}{ ZEB2 } \\
\cline { 3 - 4 } $\begin{array}{l}\text { Clinicopathological } \\
\text { factors }\end{array}$ & $\mathrm{N}$ & Positive & Negative & P-value \\
\hline Age (years) & & & & \\
$\geq 55$ & 26 & 17 & 9 & 0.825 \\
$<55$ & 24 & 14 & 10 & \\
Gender & & & & \\
Male & 28 & 18 & 10 & 0.803 \\
Female & 22 & 14 & 8 & \\
Lymph node metastasis & & & & \\
$\quad$ Yes & 34 & 23 & 11 & $\mathbf{0 . 0 3 5}$ \\
No & 16 & 5 & 11 & \\
TNM stage & & & & \\
I/II & 19 & 7 & 12 & $\mathbf{0 . 0 2 0}$ \\
III/IV & 31 & 23 & 8 & \\
\hline
\end{tabular}

Bold indicates a significant difference. ZEB2, zinc finger E-box binding homeobox 2 .

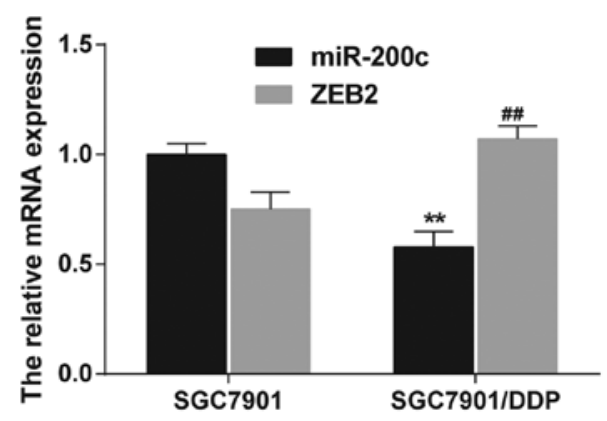

Figure 3. Relative expression of miR-200c and ZEB2 is detected by quantitative real-time RT-PCR in gastric cancer SGC7901 and SGC7901/DDP cells. The results were obtained from three independent experiments. Data are expressed as the mean $\pm \mathrm{SD} .{ }^{* *} \mathrm{P}<0.01,{ }^{\# \#} \mathrm{P}<0.01$, compared with the SGC7901 group. miR-200c, microRNA-200c; ZEB2, zinc finger E-box binding homeobox 2 .

variables among groups (mean $\pm \mathrm{SD}$ ) were compared using the procedure of analysis of variance (ANOVA) or the Student's t-test. Immunohistochemical results of the ZEB2 protein were analyzed by the Chi-squared test. A P-value $<0.05$ was defined as statistically significant.

\section{Results}

Expression levels of miR-200c and ZEB2 in gastric cancer specimens. miR-200c expression in 50 gastric cancer tissues and matched normal adjacent tissues was tested by quantitative real-time RT-PCR. Gastric cancer tissues showed a significantly downregulated miR-200c expression level when compared to that noted in the normal adjacent tissues $(\mathrm{P}<0.01$; Fig. 1). Furthermore, we analyzed the correlation between the expression of miR-200c and the pathological characteristics of gastric cancer patients. As shown in Table II, patients with 
A
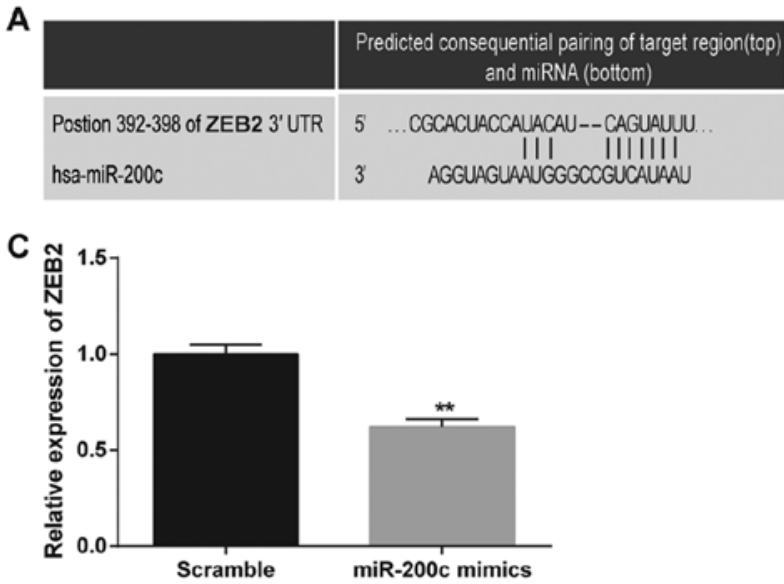

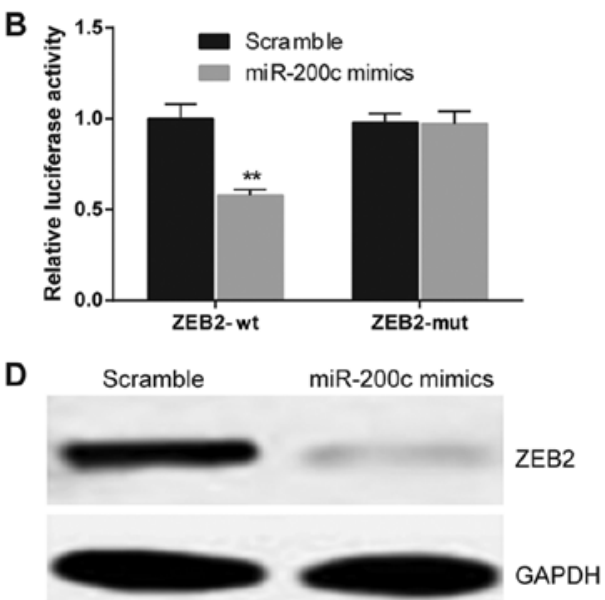

Figure 4. ZEB2 is a target gene of miR-200c. (A) Binding of miR-200c with ZEB2 3'-UTR as predicted by TargetScan. (B) Dual-luciferase reporter gene assay revealed that miR-200c significantly decreased the luciferase activity of ZEB2 wild-type (wt) 3'UTR. (C) ZEB2 mRNA levels in SGC7901/DDP cells transfected with miR-200c mimics or scramble sequence were examined by qRT-PCR. (D) The expression of ZEB2 protein was detected by western blot analysis using GAPDH as a loading control. The results were obtained from three experiments. Data are expressed as the mean $\pm \mathrm{SD}$. ${ }^{* *} \mathrm{P}<0.01$ vs. the corresponding control. ZEB2, zinc finger E-box binding homeobox 2; miR-200c, microRNA-200c.
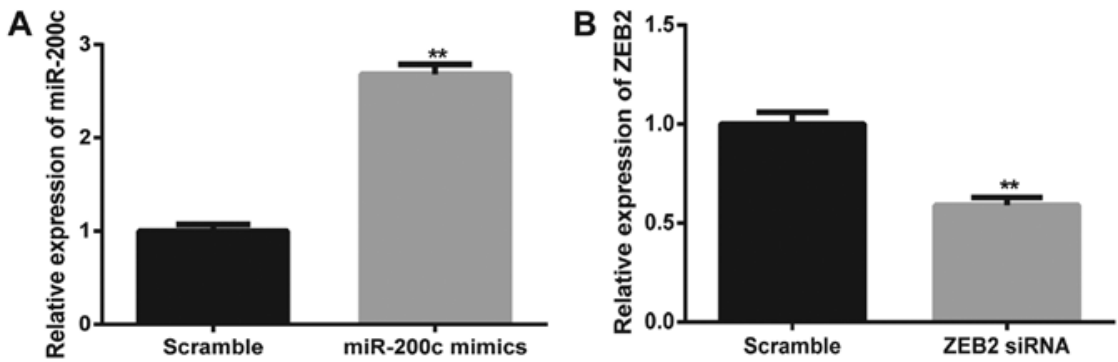

Figure 5. Expression levels of miR-200c and ZEB2 in gastric cancer SGC7901/DDP cells transfected with miR-200c mimics or ZEB2 siRNA were detected respectively by quantitative real-time RT-PCR. (A) The expression of miR-200c was significantly increased in cells transfected with miR-200c mimics. (B) The expression of ZEB2 was significantly decreased in cells transfected with ZEB2 siRNA. The results were obtained from 3 independent experiments. Data are expressed as the mean $\pm \mathrm{SD} .{ }^{* *} \mathrm{P}<0.01$, compared with the corresponding control group. miR-200c, microRNA-200c; ZEB2, zinc finger E-box binding homeobox 2 .

lymph node metastasis were associated with a marked lower expression level of miR-200c on average. In addition, patients at TNM III/IV stage had significantly lower miR-200c expression compared with patients at TNM I/II stage (all $\mathrm{P}<0.01$ ). No significant correlation was found between miR-200c expression and age or gender (both $\mathrm{P}>0.05$ ).

The protein expression of ZEB2 in gastric cancer specimens was detected by immunohistochemical method (Fig. 2). The association between the expression of ZEB2 and the clinical characteristics of the gastric cancer patients is shown in Table III. The expression of ZEB2 in patients with lymph node metastasis was significantly higher than that of patients without lymph node metastasis; the expression of ZEB2 in patients at a TNM III/IV stage was also significantly higher than that of patients at a TNM I/II stage (both $\mathrm{P}<0.05$ ). No significant association between the expression of ZEB2 and age/gender was suggested (both $\mathrm{P}>0.05$ ).

Expression of miR-200c and ZEB2 in gastric cancer SGC7901 and SGC7901/DDP cells. Results from RT-PCR revealed that the expression of miR-200c was markedly downregulated in SGC7901/DDP cells with DDP resistance compared with SGC7901 cells $(\mathrm{P}<0.01)$. Meanwhile, the expression of ZEB2 in SGC7901/DDP cells was increased in comparison to SGC7901 cells $(\mathrm{P}<0.01$; Fig. 3$)$. Therefore, we concluded that there is a potential relationship between miR-200c and ZEB2.

ZEB2 is a target gene of miR-200c. For the purpose of clarifying the potential relationship between miR-200c and ZEB2, a putative conserved binding site of miR-200c at nucleotide position 392-398 of human ZEB2 3'UTR was predicted by the TargetScan database. Perfect base pairing is shown between the seed sequence of mature miR-200c and the 3'UTR of ZEB2 mRNA (Fig. 4A). The results of the dual luciferase reporter gene assays revealed that miR-200c decreased the luciferase activity of ZEB2 wild-type by $42 \%(\mathrm{P}<0.01)$. However, the effect of miR-200c on the luciferase activity of ZEB2 with mutant-type 3'UTR was not significant (Fig. 4B). As suggested by RT-PCR and western blot analysis assays, the expression levels of both ZEB2 mRNA and protein were inhibited by the miR-200c mimics compared to the scramble group $(\mathrm{P}<0.01$; 


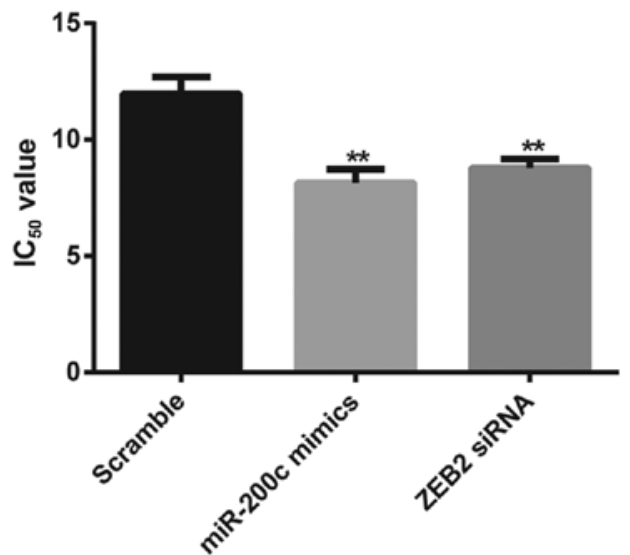

Figure 6. Regulation of miR-200c and ZEB2 expression alters the sensitivity of gastric cancer cells to DDP. SGC7901/DDP cells were transfected with miR-200c mimics or ZEB2 siRNA, after incubation with DDP for $48 \mathrm{~h}$. Cell viability was detected using the MTT method and the $\mathrm{IC}_{50}$ value of DDP was calculated. The data were obtained from 3 independent experiments. ${ }^{* *} \mathrm{P}<0.01$ compared to the scramble group. miR-200c, microRNA-200c; ZEB2, zinc finger E-box binding homeobox 2; DDP, cisplatin.
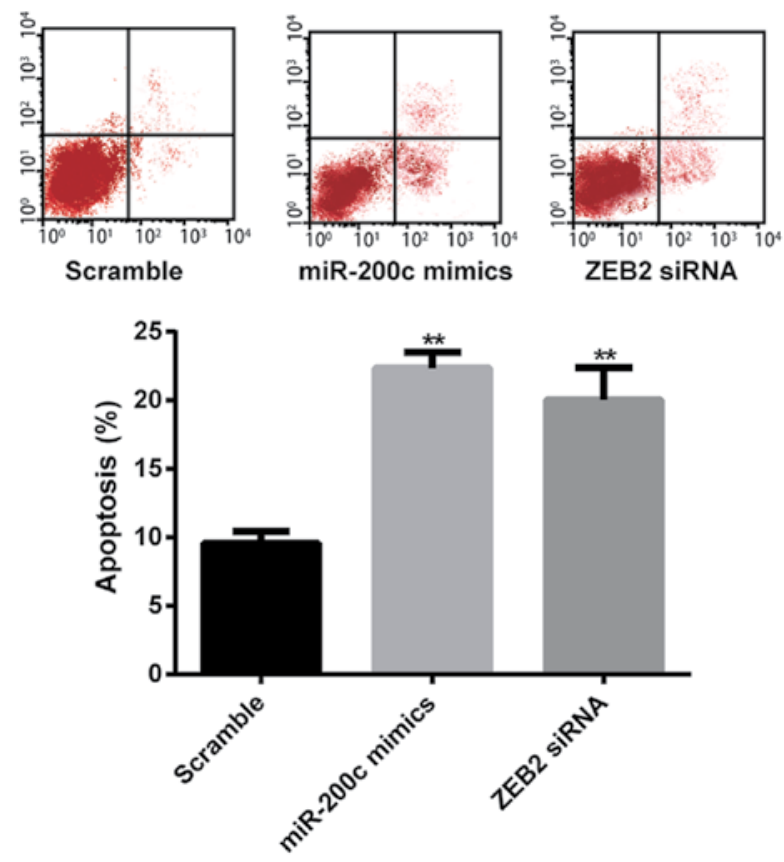

Figure 7. Overexpression of miR-200c and downregulation of ZEB2 sensitize SGC7901/DDP cells to DDP-induced apoptosis, respectively. In SGC7901/DDP cells, apoptosis assessed by flow cytometry showed a marked increase in the miR-200c mimic- and ZEB2 siRNA-transfected cells, compared with the scramble group. The data were obtained from 3 independent experiments. ${ }^{* *} \mathrm{P}<0.01$ compared to the scramble group. miR200c, microRNA-200c; ZEB2, zinc-finger E-box binding homeobox 2; DDP, cisplatin.

Fig. 4C and D). The aforementioned findings indicate that ZEB2 is a direct target of miR-200c.

Upregulation of miR-200c and knockdown of ZEB2 enhance the sensitivity of $S G C 7901 / D D P$ cells to DDP. To explore the association between miR-200c and DDP resistance in SGC7901/DDP cells, the impact of miR-200c overexpression and downregulation of ZEB2 on the DDP sensitivity of cells
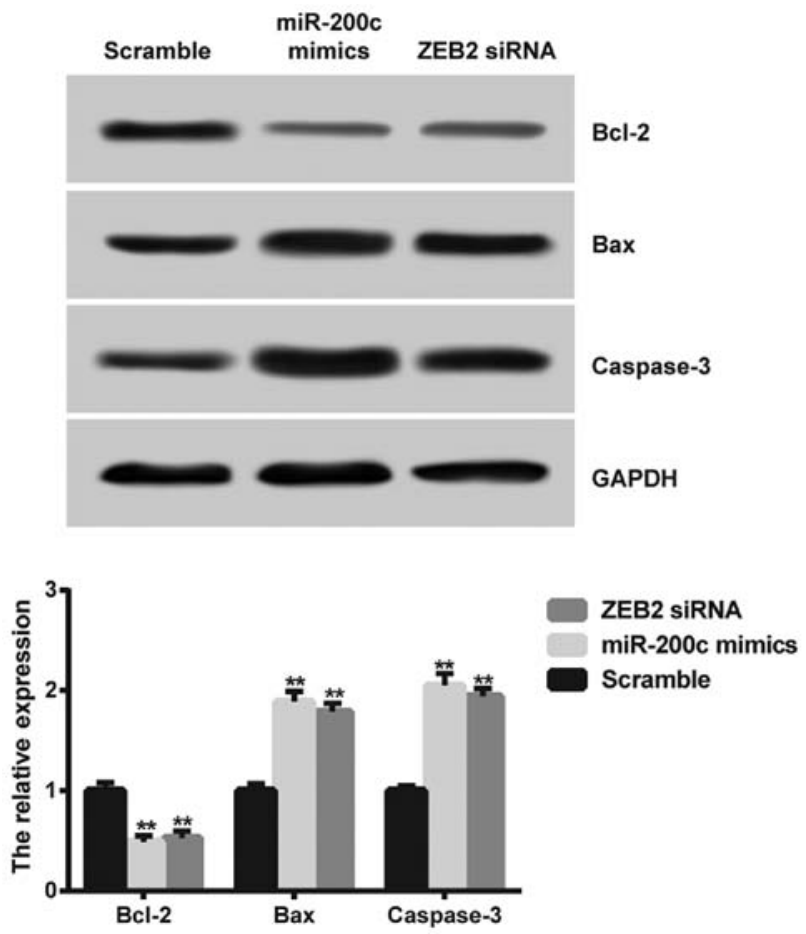

Figure 8. Alteration in the expression of $\mathrm{Bcl}-2$, Bax and caspase- 3 proteins in SGC7901/DDP cells after transfection with miR-200c mimics or ZEB2 siRNA. Results from western blot analysis showed that overexpression of miR-200c and downregulation of ZEB2 were associated with increased Bax and caspase- 3 expression and suppressed Bcl-2 expression. The data were obtained from 3 independent experiments. ${ }^{* *} \mathrm{P}<0.01$ compared to the scramble group. miR-200c, microRNA-200c; ZEB2, zinc finger E-box binding homeobox 2; DDP, cisplatin.

was assessed. The data from qRT-PCR showed that miR-200c mimics significantly increased the expression level of miR-200c, suggesting that miR-200c was efficiently transfected into the SGC7901/DDP cells (Fig. 5A). Fig. 5B shows that ZEB2 was successfully knocked down by the transfection of ZEB2 siRNA. MTT assay revealed that the SGC7901/DDP cells transfected with miR-200c mimics exhibited a significantly lower survival status than the scramble group $\left(\mathrm{IC}_{50}, 8.14 \pm 0.59\right.$ vs. $11.97 \pm 0.71 \mu \mathrm{g} / \mathrm{ml}, \mathrm{P}<0.01)$. The sensitivity of DDP was significantly enhanced in cells transfected with ZEB2 siRNA compared to the scramble group, with the $\mathrm{IC}_{50}$ of DDP at $8.78 \pm 0.39 \mu \mathrm{g} / \mathrm{ml}$ in the ZEB2 siRNA group $(\mathrm{P}<0.01$; Fig. 6$)$.

Overexpression of miR-200c and downregulation of ZEB2 sensitize cells to DDP-induced apoptosis. The results of flow cytometry showed that the SGC7901/DDP cells transfected with miR-200c mimics had a significantly higher apoptosis rate $(22.35 \pm 1.16, \mathrm{P}<0.01)$ compared with the scramble group $(9.58 \pm 0.86)$. The apoptosis rate of cells transfected with ZEB2 siRNA was also significantly increased $(20.03 \pm 2.36, \mathrm{P}<0.01)$, while any differences between the miR-200c mimics and the ZEB2 siRNA group were insignificant ( $P>0.05$; Fig. 7). These findings suggest that overexpression of miR-200c and knockdown of ZEB2 enhanced cell sensitivity to DDP and increased cell apoptosis.

Expression of apoptosis-related molecules in SGC7901/DDP cells. Western blot analysis was carried out in SGC7901/DDP 
cells to further clarify the mechanism of miR-200c and ZEB2 in regulating DDP resistance in gastric cancer. The expression levels of Bax and caspase-3 were increased in the miR-200c mimic- and the ZEB2 siRNA-transfected cells, while the expression level of Bcl-2 was markedly decreased compared to the scramble group $(\mathrm{P}<0.01$; Fig. 8$)$.

\section{Discussion}

Gastric cancer is one of the most common malignant tumors and remains the second largest threat to individuals in the world (23). The incidence of gastric cancer is higher in Eastern countries, including China, Korea, and Japan (24,25). Gastric cancer is often diagnosed at late stages in which surgical procedures may not be effective $(26,27)$. On the other hand, resistance to chemotherapy is another challenge in clinical practice (28-30). DDP is a popular chemotherapeutic medication which induces tumor cell death by DNA damage (31). Therefore, finding the related factors that may affect the resistance to DDP may improve the survival status of gastric cancer patients.

miRNAs are a class of endogenous 22-nucleotide singlestrand and highly conserved non-coding RNAs, which are forecasted to modulate about $30 \%$ of gene expression through the interference with mRNA translation (32). It has been reported that miRNAs play a multifunctional role in many biological processes including cell differentiation, apoptosis, proliferation, tumorigenesis, tumor development and tumor chemoresistance (33-38). miR-200c, which belongs to the miR-200 family, is involved in the inhibition of EMT, tumor invasion, and metastasis $(39,40)$. Many studies have shown that miR-200c can increase the sensitivity of cells to antitumor medications in a variety of cancers, including breast $(41,42)$, ovarian (43) and non-small cell lung cancer (43). In this study, miR-200c was downregulated in gastric cancer and human gastric cancer cell line SGC7901 with DDP-resistance (SGC7901/DDP cells), which is consistent with the corresponding results from a previous study (34). Therefore, all of this evidence suggests that miR-200c may be involved in regulating the chemoresistance of gastric cancer patients.

Generally, miRNAs mediate a series of biological processes through different target sites and they also regulate the expression of their downstream target mRNAs (44-46). Previous studies have identified many downstream target mRNAs of miRNA200c, such a RhoE and ZEB1/2 (34,47). Accordingly, the dual luciferase reporter gene assay in our experiments suggests that miR-200c could specifically bind with the 3'UTR of ZEB2 and significantly suppress the luciferase activity, implying that ZEB2 is a direct downstream target gene of miR-200c in SGC7901/DDP cells.

ZEB2, as a key member of the Snail gene family, is closely associated with the biological processes of numerous tumors $(48,49)$. Additionally, it has been reported that ZEB2 plays a major role in EMT by combining the E-box sequence of E-cadherin and then suppressing the transcription of numerous genes (e.g., bridge grain protein, cytokeratin and E-calcium sticky protein) (50). In our study, ZEB2 was markedly upregulated in gastric cancer tissues and SGC7901/DDP cells whereas miR-200c exhibited the opposite trend. Furthermore, our results showed that the upregulation of miR-200c or downregulation of ZEB2 could increase the sensitivity of SGC7901/DDP cells to DDP. Previous studies also suggested that upregulated miR-200c enhanced the sensitivity to chemotherapy in patients with gastric cancer $(17,34)$. Meanwhile, downregulation of ZEB2 also enhanced the sensitivity to chemotherapy in small-cell lung cancer (51). Our results also confirmed that the upregulation of miR-200c or downregulation of ZEB2 could enhance the sensitivity of SGC7901/DDP cells to DDP-induced apoptosis and therefore miR-200c and ZEB2 can potentially regulate chemotherapy sensitivity through the apoptotic signaling pathway in gastric cancer patients. Accordingly, it has been reported that miR-200c not only regulates the induction of apoptosis by targeting FAP-1 (52), but can also mediate the resistance to breast cancer medications by regulating a series of apoptosisrelated genes (53). Moreover, knockdown of ZEB2 enhanced the sensitivity of lung cancer patients to chemotherapy since chemotherapy-induced apoptosis is potentially stimulated (51).

In the present study, we demonstrated that miR-200c enhanced the sensitivity of gastric cancer to DDP by directly targeting ZEB2. However, there are still some limitations in this study. For example, only the SGC7901/DDP cell line was used in this study and the detailed molecular mechanism involved remains unclear. The molecular mechanism of miR-200c and ZEB2 with respect to DDP-induced apoptosis needs further analysis in the future. In conclusion, we assessed and reported the effect of miR-200c and its target gene ZEB2 on DDP resistance in gastric cancer. Our findings illustrated that miR200c expression was significantly downregulated in gastric cancer, while ZEB2 expression exhibited the opposite trend. Moreover, ZEB2 was found to be a direct target of miR-200c. Our data further demonstrated that upregulation of miR-200c and downregulation of ZEB2 could increase the chemotherapeutic sensitivity of gastric cancer and chemotherapy-induced apoptosis. Collectively, all of these data suggest that miR-200c enhanced the sensitivity of gastric cancer to chemotherapy by directly targeting and regulating the expression of ZEB2. Thus, both miR-200c and ZEB2 exhibit great potential to serve as effective therapeutic targets for increasing the sensitivity of gastric cancer to DDP.

\section{References}

1. Giordano A and Cito L: Advances in gastric cancer prevention. World J Clin Oncol 3: 128-136, 2012.

2. Ferlay J, Shin HR, Bray F, Forman D, Mathers C and Parkin DM: Estimates of worldwide burden of cancer in 2008: GLOBOCAN 2008. Int J Cancer 127: 2893-2917, 2010.

3. Mbulaiteye SM, Hisada M and El-Omar EM: Helicobacter pylori associated global gastric cancer burden. Front Biosci (Landmark Ed) 14: 1490-1504, 2009.

4. Lee YY and Derakhshan MH: Environmental and lifestyle risk factors of gastric cancer. Arch Iran Med 16: 358-365, 2013.

5. Schuhmacher C, Reim D and Novotny A: Neoadjuvant treatment for gastric cancer. J Gastric Cancer 13: 73-78, 2013.

6. Lordick F, Lorenzen S, Yamada Y and Ilson D: Optimal chemotherapy for advanced gastric cancer: Is there a global consensus? Gastric Cancer 17: 213-225, 2014.

7. Liu YR, Jiang YZ, Zuo WJ, Yu KD and Shao ZM: PIK3CA mutations define favorable prognostic biomarkers in operable breast cancer: A systematic review and meta-analysis. Onco Targets Ther 7: 543-552, 2014.

8. Fabian MR, Sonenberg N and Filipowicz W: Regulation of mRNA translation and stability by microRNAs. Annu Rev Biochem 79: 351-379, 2010. 
9. Bartels CL and Tsongalis GJ: MicroRNAs: Novel biomarkers for human cancer. Clin Chem 55: 623-631, 2009.

10. Ke TW, Wei PL, Yeh KT, Chen WT and Cheng YW: MiR-92a promotes cell metastasis of colorectal cancer through PTENmediated PI3K/AKT pathway. Ann Surg Oncol 22: 2649-2655, 2015.

11. Krol J, Loedige I and Filipowicz W: The widespread regulation of microRNA biogenesis, function and decay. Nat Rev Genet 11: 597-610, 2010.

12. Slabý O, Krekác D, Hrstka R, Svoboda M and Vyzula R: Involvement of microRNAs in cancer biology and possibilities of their application to diagnostic and predictive oncology. Cas Lek Cesk 147: 25-31, 2008 (In Czech).

13. Zhu W, Xu H, Zhu D, Zhi H, Wang T, Wang J, Jiang B, Shu Y and Liu P: miR-200bc/429 cluster modulates multidrug resistance of human cancer cell lines by targeting BCL2 and XIAP. Cancer Chemother Pharmacol 69: 723-731, 2012.

14. Sossey-Alaoui K, Bialkowska K and Plow EF: The miR200 family of microRNAs regulates WAVE3-dependent cancer cell invasion. J Biol Chem 284: 33019-33029, 2009.

15. Smith CM, Watson DI, Leong MP, Mayne GC, Michael MZ, Wijnhoven BP and Hussey DJ: miR-200 family expression is downregulated upon neoplastic progression of Barrett's esophagus. World J Gastroenterol 17: 1036-1044, 2011.

16. Zhang Y, Li M, Wang H, Fisher WE, Lin PH, Yao Q and Chen C: Profiling of 95 microRNAs in pancreatic cancer cell lines and surgical specimens by real-time PCR analysis. World J Surg 33: 698-709, 2009

17. Chen Y, Zuo J, Liu Y, Gao H and Liu W: Inhibitory effects of miRNA-200c on chemotherapy-resistance and cell proliferation of gastric cancer SGC7901/DDP cells. Chin J Cancer 29: $1006-1011,2010$

18. Hill L, Browne G and Tulchinsky E: ZEB/miR-200 feedback loop: At the crossroads of signal transduction in cancer. Int J Cancer 132: 745-754, 2013

19. Haslehurst AM, Koti M, Dharsee M, Nuin P, Evans K, Geraci J, Childs T, Chen J, Li J, Weberpals J, et al: EMT transcription factors snail and slug directly contribute to cisplatin resistance in ovarian cancer. BMC Cancer 12: 91, 2012.

20. Adam L, Zhong M, Choi W, Qi W, Nicoloso M, Arora A Calin G, Wang H, Siefker-Radtke A, McConkey D, et al: miR-200 expression regulates epithelial-to-mesenchymal transition in bladder cancer cells and reverses resistance to epidermal growth factor receptor therapy. Clin Cancer Res 15 5060-5072, 2009

21. Washington $\mathrm{K}: 7$ th edition of the AJCC cancer staging manual: stomach. Ann Surg Oncol 17: 3077-3079, 2010

22. Yamaue H, Tanimura H, Noguchi K, Hotta T, Tani M, Tsunoda T, Iwahashi M, Tamai $\mathbf{M}$ and Iwakura S: Chemosensitivity testing of fresh human gastric cancer with highly purified tumour cells using the MTT assay. Br J Cancer 66: 794-799, 1992.

23. Kelley JR and Duggan JM: Gastric cancer epidemiology and risk factors. J Clin Epidemiol 56: 1-9, 2003.

24. Lee JH, Kim KM, Cheong JH and Noh SH: Current management and future strategies of gastric cancer. Yonsei Med J 53: 248-257, 2012.

25. Hudler P: Genetic aspects of gastric cancer instability. ScientificWorldJournal 2012: 761909, 2012

26. Yan LH, Wei WY, Cao WL, Zhang XS, Xie YB and Xiao Q Overexpression of E2F1 in human gastric carcinoma is involved in anti-cancer drug resistance. BMC Cancer 14: 904, 2014.

27. Kang JH, Lee SI, Lim DH, Park KW, Oh SY, Kwon HC, Hwang IG, Lee SC, Nam E, Shin DB, et al: Salvage chemotherapy for pretreated gastric cancer: A randomized phase III trial comparing chemotherapy plus best supportive care with best supportive care alone. J Clin Oncol 30: 1513-1518, 2012.

28. Sun F, Lu X, Li H, Peng Z, Wu K, Wang G and Tong Q: Special AT-rich sequence binding protein 1 regulates the multidrug resistance and invasion of human gastric cancer cells. Oncol Lett 4: 156-162, 2012.

29. Zhang H, Sun LL, Meng YL, Song GY, Hu JJ, Lu P and Ji B: Survival trends in gastric cancer patients of Northeast China. World J Gastroenterol 17: 3257-3262, 2011

30. Maconi G, Manes G and Porro GB: Role of symptoms in diagnosis and outcome of gastric cancer. World J Gastroenterol 14: 1149-1155, 2008.

31. Montagnani F, Turrisi G, Marinozzi C, Aliberti C and Fiorentini G: Effectiveness and safety of oxaliplatin compared to cisplatin for advanced, unresectable gastric cancer: a systematic review and meta-analysis. Gastric Cancer 14: 50-55, 2011.
32. Guo H, Ingolia NT, Weissman JS and Bartel DP: Mammalian microRNAs predominantly act to decrease target mRNA levels. Nature 466: 835-840, 2010

33. Wang J, Yang M, Li Y and Han B: The role of microRNAs in the chemoresistance of breast cancer. Drug Dev Res 76: 368-374, 2015.

34. Chang L, Guo F, Wang Y, Lv Y, Huo B, Wang L and Liu W: MicroRNA-200c regulates the sensitivity of chemotherapy of gastric cancer SGC7901/DDP cells by directly targeting RhoE. Pathol Oncol Res 20: 93-98, 2014.

35. Issabekova A, Berillo $\mathrm{O}$, Regnier $\mathrm{M}$ and Anatoly I: Interactions of intergenic microRNAs with mRNAs of genes involved in carcinogenesis. Bioinformation 8: 513-518, 2012.

36. Hong L, Han Y, Lu Q, Zhang H, Zhao Q, Wu K and Fan D: Drug resistance-related microRNAs in esophageal cancer. Expert Opin Biol Ther 12: 1487-1494, 2012.

37. Sato F, Tsuchiya S, Meltzer SJ and Shimizu K: MicroRNAs and epigenetics. FEBS J 278: 1598-1609, 2011.

38. Huang Y, Shen XJ, Zou Q, Wang SP, Tang SM and Zhang GZ: Biological functions of microRNAs: A review. J Physiol Biochem 67: 129-139, 2011.

39. Korpal M and Kang Y: The emerging role of miR-200 family of microRNAs in epithelial-mesenchymal transition and cancer metastasis. RNA Biol 5: 115-119, 2008.

40. Hurteau GJ, Carlson JA, Spivack SD and Brock GJ: Overexpression of the microRNA $h s a-m i R-200 \mathrm{c}$ leads to reduced expression of transcription factor 8 and increased expression of E-cadherin. Cancer Res 67: 7972-7976, 2007.

41. Knezevic J, Pfefferle AD, Petrovic I, Greene SB, Perou CM and Rosen JM: Expression of miR-200c in claudin-low breast cancer alters stem cell functionality, enhances chemosensitivity and reduces metastatic potential. Oncogene 34: 5997-6006, 2015.

42. Bai WD, Ye XM, Zhang MY, Zhu HY, Xi WJ, Huang X, Zhao J, Gu B, Zheng GX, Yang AG, et al: MiR-200c suppresses TGF- $\beta$ signaling and counteracts trastuzumab resistance and metastasis by targeting ZNF217 and ZEB1 in breast cancer. Int J Cancer 135: 1356-1368, 2014

43. Liu N, Zeng J, Zhang X, Yang Q, Liao D, Chen G and Wang Y: Involvement of miR-200a in chemosensitivity regulation of ovarian cancer. Zhonghua Yi Xue Za Zhi 94: 2148-2151, 2014 (In Chinese).

44. Zheng L, Zhang Y, Liu Y, Zhou M, Lu Y, Yuan L, Zhang C, Hong M, Wang S and Li X: MiR-106b induces cell radioresistance via the PTEN/PI3K/AKT pathways and $\mathrm{p} 21$ in colorectal cancer. J Transl Med 13: 252, 2015.

45. Ritchie W, Rasko JE and Flamant S: MicroRNA target prediction and validation. Adv Exp Med Biol 774: 39-53, 2013.

46. Witkos TM, Koscianska E and Krzyzosiak WJ: Practical aspects of microRNA target prediction. Curr Mol Med 11: 93-109, 2011.

47. Panda H, Pelakh L, Chuang TD, Luo X, Bukulmez $\mathrm{O}$ and Chegini N: Endometrial miR-200c is altered during transformation into cancerous states and targets the expression of ZEBs, VEGFA, FLT1, IKK $\beta, K L F 9$, and FBLN5. Reprod Sci 19: 786-796, 2012

48. Lee JY, Park MK, Park JH, Lee HJ, Shin DH, Kang Y, Lee CH and Kong G: Loss of the polycomb protein Mel-18 enhances the epithelial-mesenchymal transition by ZEB1 and ZEB2 expression through the downregulation of miR-205 in breast cancer. Oncogene 33: 1325-1335, 2014

49. Nam EH, Lee Y, Park YK, Lee JW and Kim S: ZEB2 upregulates integrin $\alpha 5$ expression through cooperation with $\mathrm{Sp1}$ to induce invasion during epithelial-mesenchymal transition of human cancer cells. Carcinogenesis 33: 563-571, 2012.

50. Kakihana M, Ohira T, Chan D, Webster RB, Kato H, Drabkin HA and Gemmill RM: Induction of E-cadherin in lung cancer and interaction with growth suppression by histone deacetylase inhibition. J Thorac Oncol 4: 1455-1465, 2009.

51. Fang S, Zeng X, Zhu W, Tang R, Chao Y and Guo L: Zinc finger E-box-binding homeobox 2 (ZEB2) regulated by miR-200b contributes to multi-drug resistance of small cell lung cancer. Exp Mol Pathol 96: 438-444, 2014.

52. Schickel R, Park SM, Murmann AE and Peter ME: miR-200c regulates induction of apoptosis through CD95 by targeting FAP-1. Mol Cell 38: 908-915, 2010.

53. Tryndyak VP, Beland FA and Pogribny IP: E-cadherin transcriptional down-regulation by epigenetic and microRNA-200 family alterations is related to mesenchymal and drug-resistant phenotypes in human breast cancer cells. Int J Cancer 126: 2575-2583, 2010. 